\title{
ANALYSIS OF HUMAN FACE RECOGNITION ALGORITHM USING PCA+FDIT IN IMAGE DATABASE FOR CRIME INVESTIGATION
}

\author{
V.S. MANJULA, \\ Asst. Professor, \\ Dept. of Computer Application, \\ St. Joseph' College, Chennai- 600122 , INDIA. \\ manjusunil.vs@gmail.com
}

\section{ABSTRACT:}

In general, the field of face recognition has lots of research that have put interest in order to detect the face and to identify it and also to track it. Many researchers have concentrated on the face identification and detection problem by using various approaches. The proposed approach is further very useful and helpful in real time application. Thus the Face Detection, Identification which is proposed here is used to detect the faces in videos in the real time application by using the FDIT (Face Detection Identification Technique) algorithm. Thus the proposed mechanism is very help full in identifying individual persons who are been involved in the action of robbery, murder cases and terror activities. Although in face recognition the algorithm used is of histogram equalization combined with Back propagation neural network in which we recognize an unknown test image by comparing it with the known training set images that are been stored in the database. Also the proposed approach uses skin color extraction as a parameter for face detection. A multi linear training and rectangular face feature extraction are done for training, identifying and detecting. Thus the proposed technique is PCA + FDIT technique configuration only improved recognition for subjects in images are included in the training data. It is very useful in identify a proposed technique is PCA + FDIT technique configuration only improved recognition for subjects in images are included in the training data. It is very useful in identify a
single person from a group of faces. Thus the proposed technique is well suited for all kinds faces frame work for face detection and identification. The face detection and identification modules share the same hierarchical architecture. They both consist of two layers of classifiers, a layer with a set of component classifiers and a layer with a single combination classifier. Also we have taken a real life example and simulated the algorithms in IDL Tool successfully.

1.

Keywords: PCA, PRDIT, FDIT, histogram equalization, rectangular features.

\section{INTRODUCTION:}

The face is our primary focus of attention in social life playing an important role in conveying identity and emotions. Detection and recognisation of face is an important research in the area of computer vision [1]. The process of face detecting is a challenge and toughest process because of different facial expression, races, backgrounds, illumination, overlapping, low brightness make the face detection process as more complicated one.

Computational models of face recognition are interesting because they can contribute not only to theoretical knowledge but also to practical applications. Computers that detect and recognize faces could be applied to a wide variety of tasks including criminal identification, security system, image and film processing, identity verification, tagging purposes and human-computer interaction. Unfortunately, developing a computational model of face detection and recognition is quite difficult because faces are complex, multidimensional and meaningful visual stimuli [2].

Thus the Face detections are used in many places now a days especially on websites hosting like picassa and face book. The automatically tagging feature adds a new dimension in order to share pictures among the people who are in the picture and also gives idea to other people about who the person in the image. Here we have studied and implemented a pretty simple but very effective face detection algorithm which takes human skin colour into account for detecting and tracking the face[3][4].

Also here we propose a new approach which is based on the multi linear training and rectangular face feature extraction. Detecting, training, tracking and identification are the major steps of the proposed technique. The main aim, which we believe we have reached, was to develop a method of face recognition that is of fast, robust, reasonably simple and accurate with a relatively simple and easy to understand algorithms and techniques [4].

\section{PERSPECTIVE OF OUR WORK:}

The proposed approach is simple, fast and accurate which is been applied together as a single algorithm to provide better results under complex circumstances like face position, luminance variation etc. Each of these algorithms are been discussed one by one below. Thus the proposed approach handle changes on the face image like lighting, complexity in the background, multiple faces in the image. Thus the proposed approach makes an improvement in the detection results rather than the other detectors. The more challenging function in the detectors is to handle the poses. Different type of pose makes conflict in detecting a particular personality and our proposed approaches overcome these drawbacks [1]. Next the various method of tracking approach gets confused in the beginning stage and we use a tracker algorithm for the detection and it avoids the above mentioned problem. The information for the detecting process incorporates with the parameter and reproduces the information itself. Hence these algorithms always find faces in the frames even though the frame based detectors gets fails. Thus the knowledge of training can understand new faces that are entered in the training and it is always ready to integrate in the updating process [7].

$788 \mid \mathrm{P}$ a g e

W w w i j cton 1 in e.com 


\subsection{TRAINING PHASE:}

The training process is done by applying a back-propagation neural network. The input will be of face features along with that the individual users' names are fed into the system for processing. In order to extract the skin color from the face images we apply a histogram equalization process. Thus the trained data hold individual users skin color and some extra facial features. Also here we introduce a new proposed rectangle based approach for detecting the face [8]. In this approach the human face images are been splitted into various rectangle portions with different shapes and size also with their different features are also been saved along with the training samples in databases for the later verification. Figure 1.explains about the training procedure of our proposed approach.
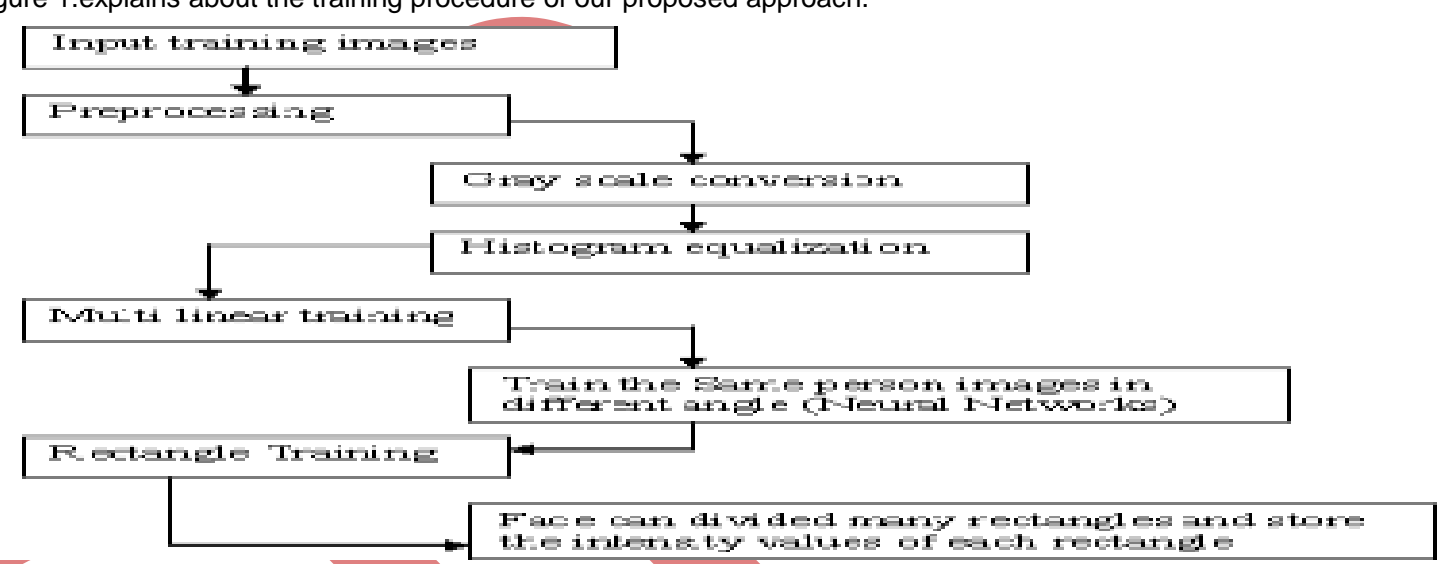

\section{Figure 1.Trainning Module}

For training the database 100 input face images with different features are given as input to the training system. The input images are taken from GTAV Face Database for training the input datasets [10]. The face and non-face block weights are calculated as negative and positive values for the neural network.

\subsection{Preprocessing of the Image:}

Face images are given as input to the proposed system which extracts multi features of the face. Also various face regions are taken under some conditions e.g. background, light and distance between the camera and face makes the different appearance of the face in the image. For getting the scale variance property, the input images where resized as pyramid image. Also we partition the image as $21 \times 21$ pixel blocks which is present in the sub-sampled image and then normalize the blocks of the image to unit variance and zero means.

\section{We take an $R G B$ color image as input}

And produce a grayscale image as output

\section{$\left(B_{i}, G_{i}, B_{i}\right) \in[0.1]^{3}$}

$T \in[0.1]$

\section{To avoid gamma correction issues.}

\subsubsection{Mathematical approach}

Apply the same method individually to the RGB image i.e., red, green and blue components in the color model. Applying the similar color method to the $\mathrm{RGB}$ image may produce the different color balance in the images. Since the color channels changes their algorithm for the getting the relative distribution. First of all the image is changed to another space color like HSV/HSL or Lab color space then we apply the proposed algorithm to value channels or luminance which does not affect the image hue and saturation.

Let us take the image of discrete grayscale and consider $n_{i}$ be the gray level occurrence number $i$. 
Council for Innovative Research www.cirworld.com
International Journal of Computers \& Technology

Volume 4 No. 3, March-April, 2013, ISSN 2277-3061

The probability of an occurrence of a pixel of level $i$ in the image is

Where, $i \in 0 \ldots L-1$

$L \rightarrow$ gray level total number in the image.

$n \rightarrow$ pixels total number of the image

$P \rightarrow$ in fact the image's histogram and Normalized to $[0,1]$

$x \rightarrow$ an occurrence of a pixel in the image

Where $\boldsymbol{C} \rightarrow$ the cumulative distribution function corresponding to $p$,

To produce the original image of level $y$ on the original image level on $x$ we have to transform the form .i.e Y's cumulative probability function be the linearized in the range of value.

The transformation is defined by:

$\mathrm{Y}_{\mathrm{i}} \rightarrow$ linearized transformation of pixels

Note that the $T$ plots the levels between the domain of $0 . . .1$. to plot the values in the original domain The following transformation should be applied to the results.

$y_{i}^{\prime} \rightarrow$ equalized image

Max $\rightarrow$ Maximam value pixel in the image

Min $\rightarrow$ Minmam value pixel in the image

\subsubsection{GABOR FILTER BASED NEURAL NETWORK:}

Face recognition is achieved by employing a multilayer perceptron with backpropagation algorithm. The architecture of the neural network is illustrated. The first layer called Gabor layer, receives the Gabor features. The number of nodes in this layer is, obviously equal to the dimension of the feature vector incorporating the Gabor features. The number of nodes in the output layer equals to the number of individual faces the network is required to recognize. The number of epochs for this experiment was 10,000 and the goal was 0.01. The back-propagation training algorithm is illustrated in a flowchart as shown in Fig.3.1.

\section{FDIT APPROACH:}

Face Detection Identification and Tracking (FDIT) technique is an extension of PRDIT that uses histogram equalization process and skin color extraction and neura network with the proficient of learning the interactions of the multiple factors like different viewpoints, different lighting conditions, different expressions etc. In the face detection process skin color plays an important role when extracting the information in the area of face [8]. That obtained color information is used to make the task easier. The process of identifying the face localization is very hard to achieve but the color information makes it easier in the complex environment. The researchers offer the many techniques for detecting the skin information. Generally no one have the same skin information. The color of the skin is differed in each person. It is arranged in the small portion of area in $\mathrm{CbCr}$ plane [9]. This model is the strong as compared with the other model having the skin information. The people in the different areas like Europe, Africa and Asia has the different type of skin in formation. So we implement the YCbCr color model to detect the color of the skin [10].

\subsection{Detection of the Face}

We randomly provide the image input to find whether the human being face is present or not in the input photos or videos. If the face is present, it return the location and the degree of the space between each face.

GRect $_{\mathrm{si}}=$ GRect $_{\mathrm{f}}\left(\mathrm{l}_{\mathrm{i}}>\right.$ equalized value)

$$
\text { SRect }_{s i}=\text { GRect }_{s i}\left(w^{\star} h\right) / n_{i}
$$


Council for Innovative Research www.cirworld.com
International Journal of Computers \& Technology

Volume 4 No. 3, March-April, 2013, ISSN 2277-3061

If $I_{i}\left(S R e c t_{s i}\right)=$ Train2

This rectangle is in face

Else

This rectangle not in face

Where

GRect $_{\text {si }} \rightarrow$ Face rectangle Group image

GRect $_{f} \rightarrow$ Full input Group mage Rectangle

SRect $_{\text {si }} \rightarrow$ Small rectangle Group image
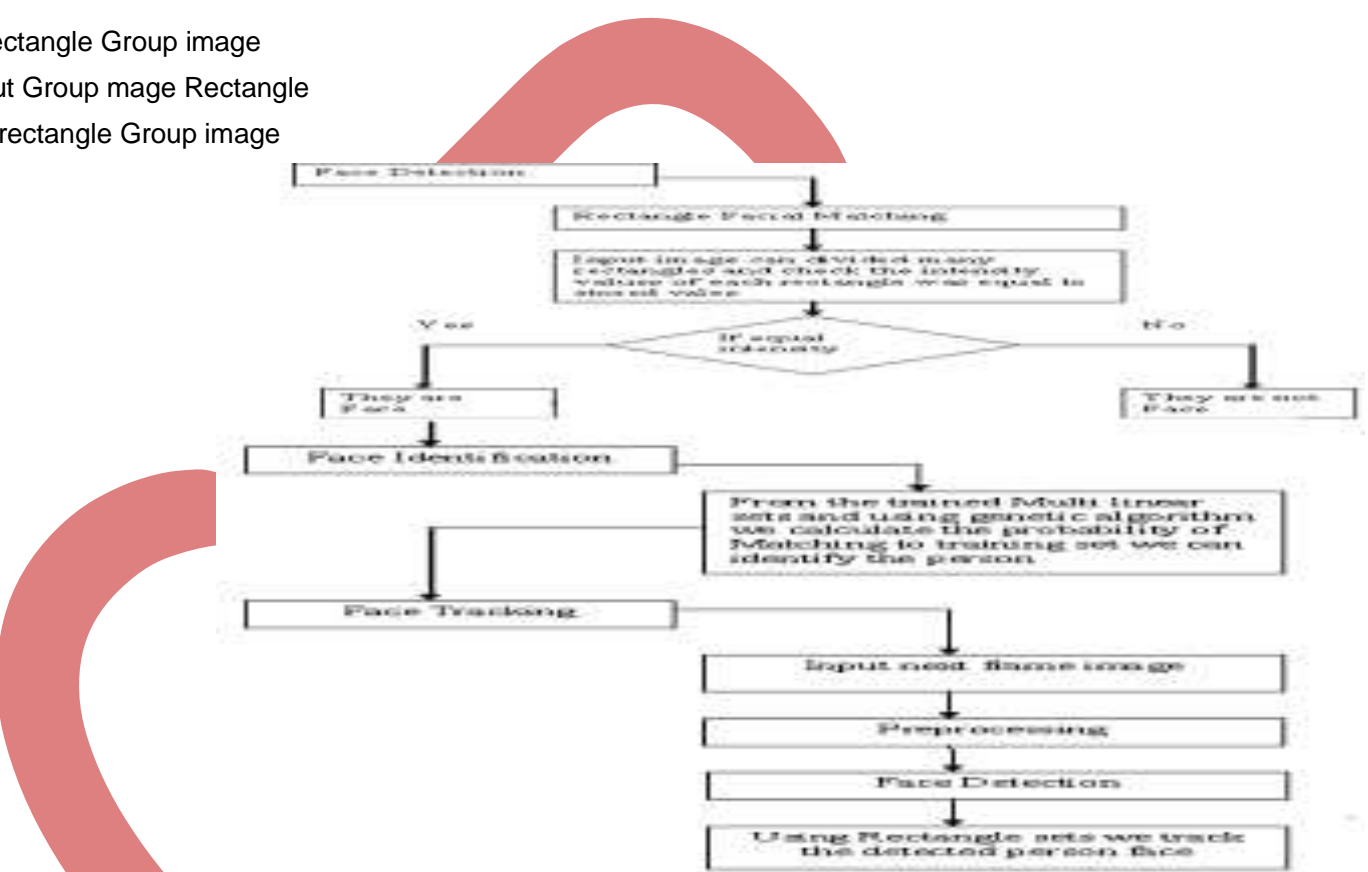

\subsection{Identification of the Face:}

All face images are divided into blocks of triangles which are providing to the neural network. Some of the face candidate blocks will be in the output of the networks. To eliminate the false detection blocks we present the face verification methods. The basic idea behind in this concept is considering the edge point distribution in facial features. By using the method of the blocks of the each candidates the edge map detecting is done. Then the proposed algorithm stores the features of the face rectangle for the various images. The images which are stored in the databases will be matched with the any of the features of the input images.

\section{If $I_{i}\left(\right.$ GRect $\left._{\text {si }}\right)=$ Train1}

\section{Face Identified who is that person}

\section{STRect $=$ GRect}

Else

\section{No one Identified}

Where

STRect $\rightarrow$ Store the Identified face rectangle 


\subsection{Face Tracking \& Recognisation:} categories.

In the active scenario, the tracking is the more important process and it follows the sequence of process. Also the process of tracking is divided into two

\section{1) Head tracking}

2) Facial feature tracking

Head tracking methods is color, region and shape based one and the feature tracking method has trackers for each and every feature and outlines the points like mouth, eyes for the tracking process. For this process of predicting and updating Condensation filter and Kalman filter are used. The grouping of the head tracking and facial feature tracking along with the concepts of filter reduce the overall problem. The framework mainly focused to watch out the multiple faces at same time. By using the Kalman filters the process of tracking of multiple people were performed.

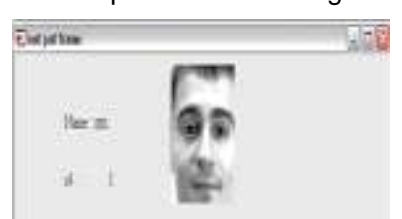

\section{If $I_{i}\left(\right.$ NFRect $\left._{s i}\right)=$ STRect} Identified person was present in the frame

\section{Else}

\section{Identified person was not present in the frame}

Where

$N F \rightarrow$ Next Frame of Group image (applied preprocessing and Face Detection)

NFRect $_{s i} \rightarrow$ Next Frame Face rectangles

\section{RESULTS AND DISCUSSION:}

To deliver the effective system we take the various color images which includes many faces with the numerous size and have the dissimilar lighting circumstance and different expressions. The color of the skin may be varied because of the different

lighting effect. To minimize the lighting effects we include the gray methods which carry out the damages in the light effects. Then the color components of RGB color i.e., Red, Green and Blue nonlinearly changed to the YCbCr color space.

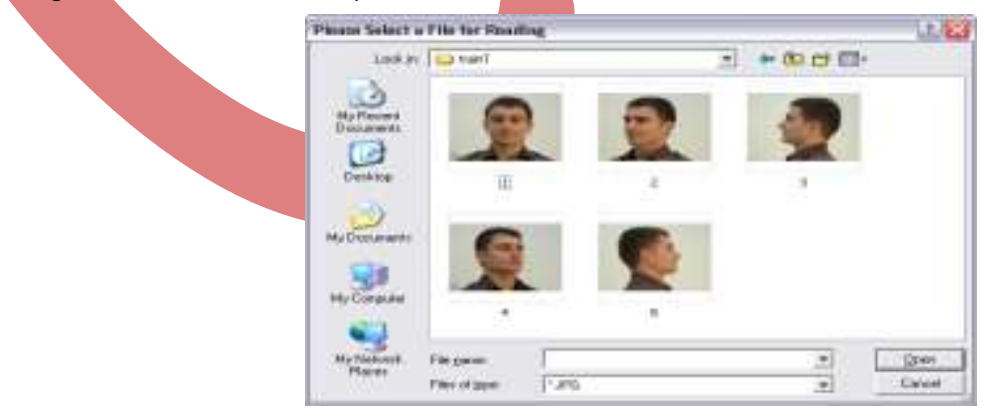

Figure 5.1 training data sets 
The databases (6406 faces) described in the previous section were split in a set of training data and a set of testing data. To deliver the effective system we take the various color images which includes many faces with the numerous size and have the dissimilar lighting circumstance and different expressions. The color of the skin may be varied because of the different lighting effect. To minimize the lighting effects we include the gray methods which carry out the damages in the light effects.

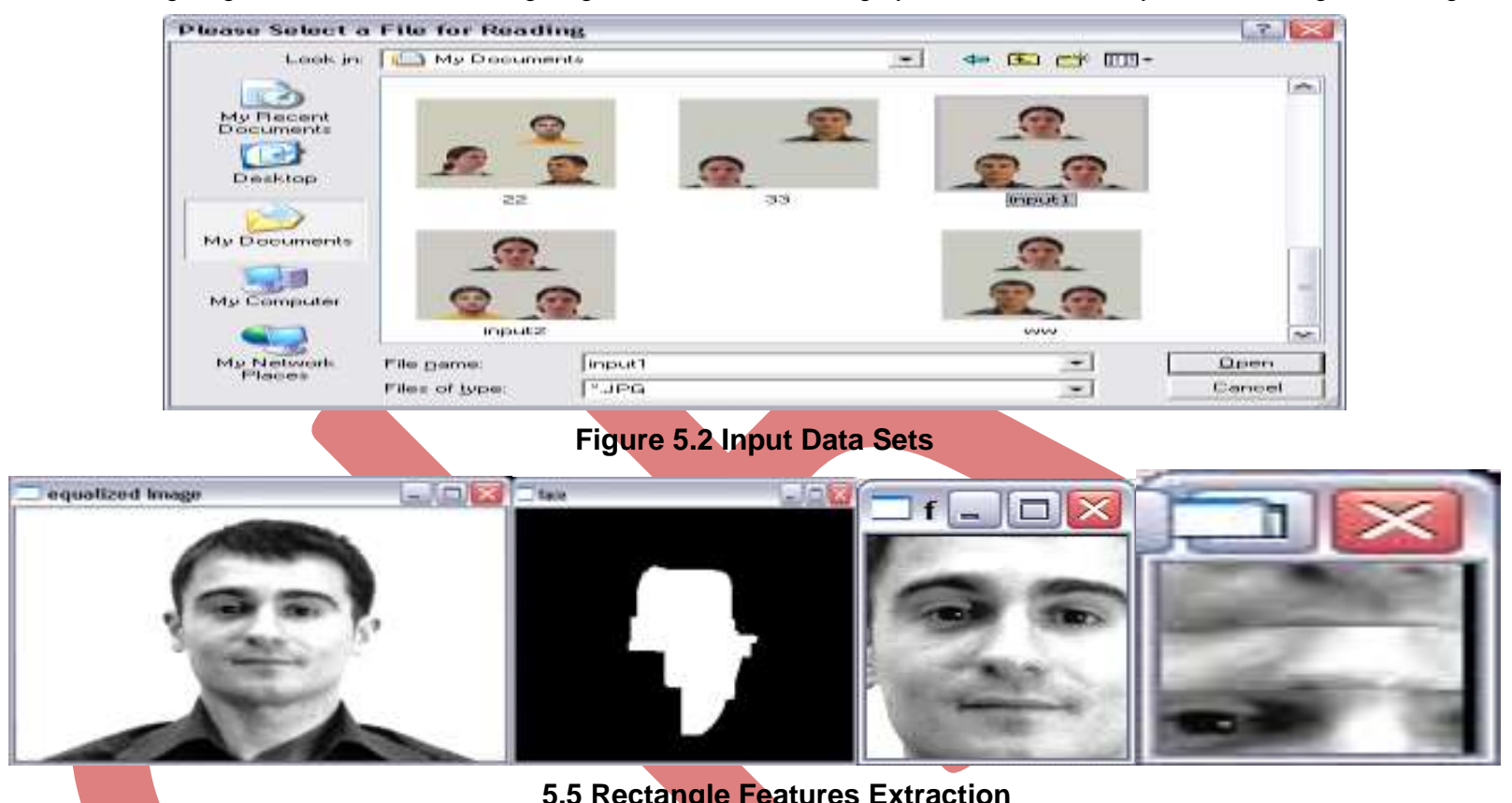

An intuitive choice of components for face recognition is the eyes, the nose and the mouth. However, it is not clear what exactly the size and shape of these components should be and whether there are other components which are equally important for recognition. Furthermore, we would like to quantify the discriminatory power of each

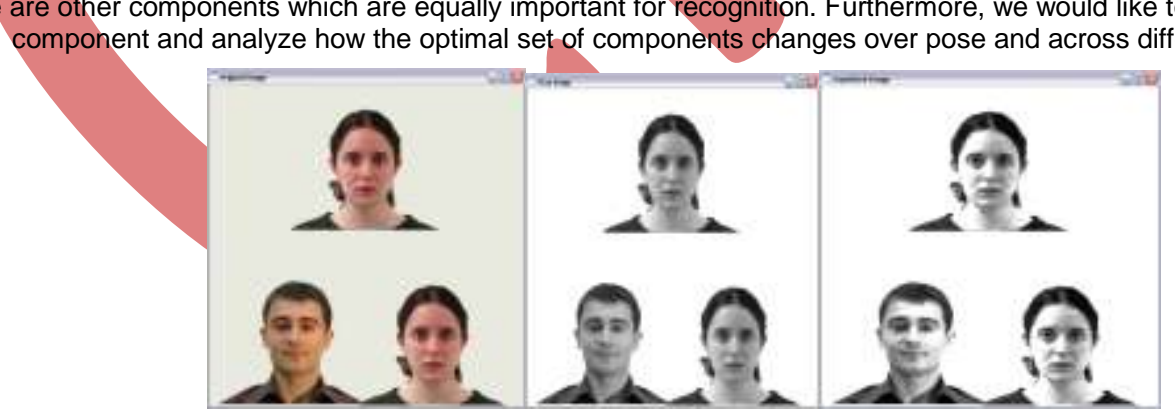

Figure 5.3 Gray Scale Image 


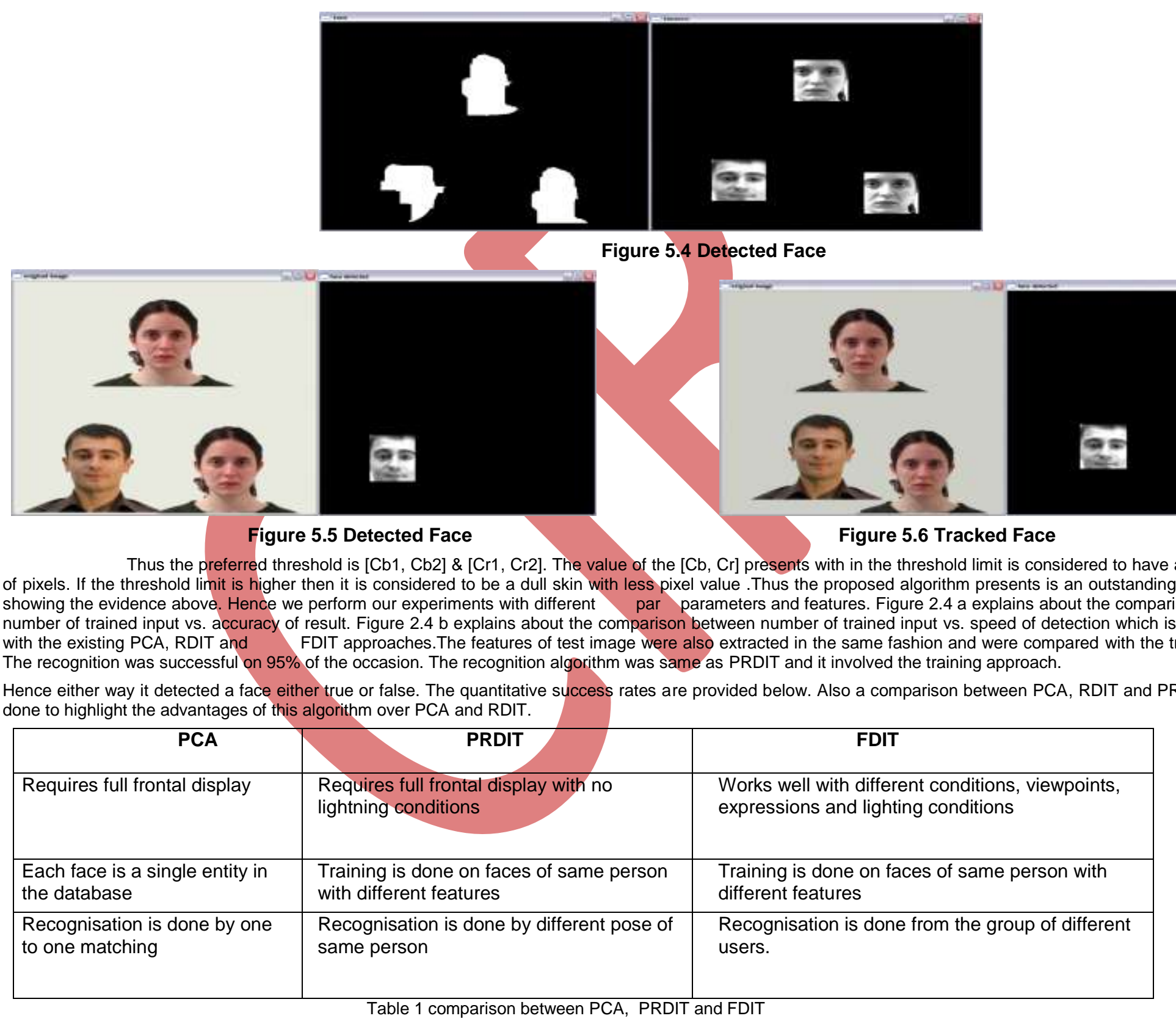


Thus these proposed techniques works well under any robust conditions like complex background and also with different face positions. These algorithms give different rates of accuracy under different conditions as experimentally observed.

A test e shown in figure 2.5a was taken on the training of different of different types of technique versus accuracy in percentage were highlighted.

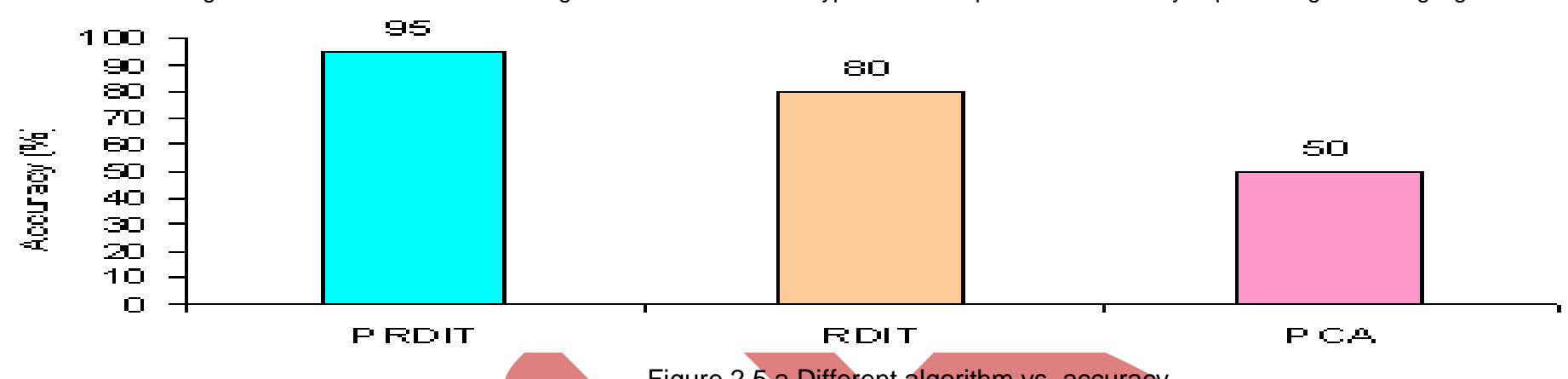

Figure 2.5 a Different algorithm vs. accuracy

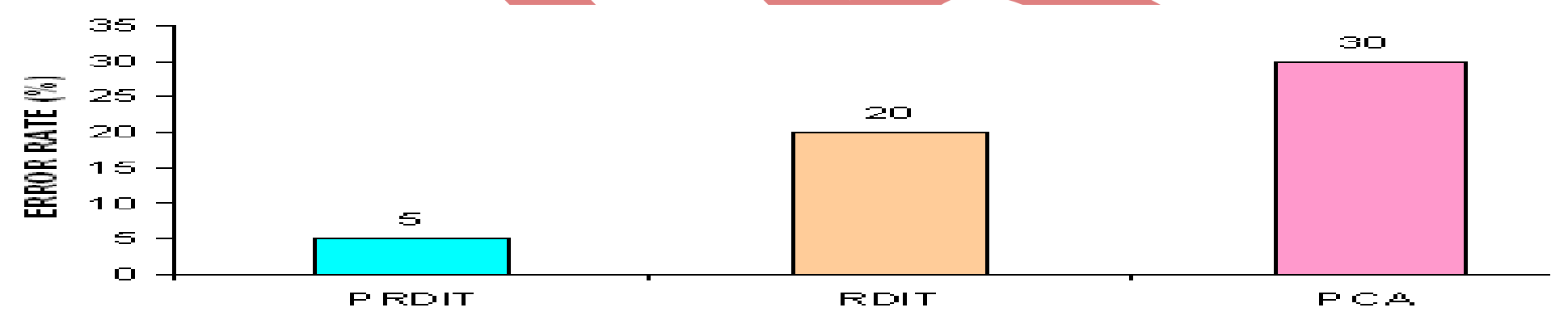

\begin{tabular}{|l|c|}
\hline \multicolumn{1}{|c|}{ Figure 2.5 b Different algorithm vs. Error Rates } \\
\hline \multicolumn{1}{|c|}{ Methods } & Recognition Rates \\
\hline Markov Random Fields & 95.91 \\
\hline $\begin{array}{l}\text { Guided Particle Swarm } \\
\text { Optimization }\end{array}$ & 93.21 \\
\hline $\begin{array}{l}\text { Regularized Discriminant } \\
\text { Analysis based Booting }\end{array}$ & 96.51 \\
\hline Proposed Method & 97.76 \\
\hline
\end{tabular}

Figure 5.7 
Comparison of facial expression recognition using image database

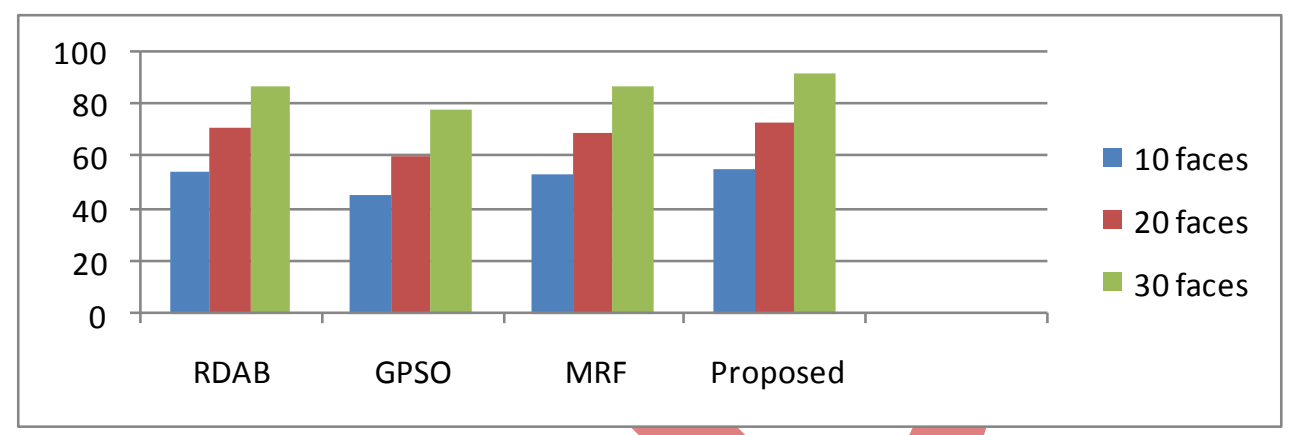

The above results are shown as comparison graphs in the following

The experiment was conducted again and again which provided a $100 \%$ result. Also the experiment was proved in Figure $2.5 \mathrm{~b}$ that the proposed algorithm has a tolerable Error rates.

\section{CONCLUSION:}

Face Deduction is to extract the face features and to deduct the features in small size of rectangle to calculate the intensity values(face size variation) and using histogram to equalize the all values are equals and to apply this method to match and to improve the accuracy level increased. This is existing technique we are applying multilinear technique to find different position training and different angle to find the person.

In single person's face we are applying rectangle feature method and multilinear technique to calculate the different person's skin color and different pose also to calculate the single persons face. It will be improving the intensity value to be increased. . The RDIT, PCA and FDIT success rates were given for face detection and the success rate was different for different images depending on the external factors. The overall success rate was $95 \%$.

\section{REFERENCES:}

1. Chiang.C.C et al., "A novel method for detecting lips eyes and faces in real time", Real Time Imaging-2003

2. Garcia.C \& Tziritas.G "Face detection using quantized skin color regions merging and wavelet packet analysis", IEEE Trans. Multimedia-1999

3. Guetta.A \& Rajagopal.s and Pare.M "Face Detection EE368 Final Project"- 2003

4. HsuR.L. \& Jain.A.K and Abdel Mottaleb.M "Face detection in color images", IEEE Trans-2002

5. Kruppa.H \& Schiele.B "Using Local Context to Improve Face Detection", In Proc. of the British Machine Vision Conference (BMVC), Norwich-2003

6. Mostafa.L \& Abdelazeem.S "Face Detection based on Skin Color using Neural Networks", in Proc. of $1^{\text {st }}$ ICGST Intl Conference on Graphics, Vision and Image Processing GVIP, Egypt-2005

7. Pitas.I \& Karasaridis.A "Multichannel transforms for signal image processing" IEEE Trans Image Processing-1996

8.Rizzi.A \& Marini.D and Gatta.C "Color correction between gray world and white patch", The human Vision and Electronic Imaging Conf-2002

9.Viola.P \& Jones.M.J "Robust real time face detection", Intl Journal of Computer Vision-2004

10.Wang.H \& Chang.S.F "A highly efficient system for automatic face region detection in MPEG videos", IEEE Trans-Circuit Systems for Video Technology in 1997

11. Yepeng Guan \& Lin Yang "unsupervised face detection based on skin color and geometric information" 